\title{
The number of planar graphs and properties of random planar graphs
}

\author{
Omer Giménez ${ }^{\dagger}$ and Marc Noy \\ Departament de Matemàtica Aplicada II, Universitat Politècnica de Catalunya \\ Jordi Girona 1-3, 08034 Barcelona, Spain \\ \{omer.gimenez, marc.noy\}@upc.edu
}

We show an asymptotic estimate for the number of labelled planar graphs on $n$ vertices. We also find limit laws for the number of edges, the number of connected components, and other parameters in random planar graphs.

Keywords: Planar graph, random graph, asymptotic enumeration, limit law, normal law, analytic combinatorics.

\section{Introduction}

The goal of this paper is to determine the asymptotic number of labelled planar graphs and to establish limit laws of random labelled planar graphs. From now on, unless stated otherwise, all graphs are labelled.

Recall that a graph is planar if it admits an embedding in the sphere. We remark that we consider planar graphs as combinatorial objects, without referring to a particular topological embedding.

Let $g_{n}$ be the number of planar graphs on $n$ vertices. A superadditivity argument [10] shows that the following limit exists:

$$
\gamma=\lim _{n \rightarrow \infty}\left(g_{n} / n !\right)^{1 / n}
$$

Until recently, the constant $\gamma$ was known only within certain bounds, namely

$$
26.18<\gamma<30.06
$$

The lower bound results from the work of Bender, Gao and Wormald [1]. They show that, if $b_{n}$ is the number of 2-connected planar graphs, then

$$
\lim _{n \rightarrow \infty}\left(b_{n} / n !\right)^{1 / n} \approx 26.18
$$

Hence $\gamma$ is at least this value.

The upper bound is based on the fact that an unlabelled planar graph on $n$ vertices can be encoded with at most $\alpha n$ bits for some constant $\alpha$. If this is the case then $g_{n} \leq 2^{\alpha n} n$ !, and so $\gamma \leq 2^{\alpha}$. The first such result was obtained by Turán [14] with the value $\alpha=12$. This has been improved over the years and presently the best result is $\alpha \approx 4.91$, obtained by Bonichon et al. [2]. Since $2^{4.91} \approx 30.06$, the upper bound follows.

Recently the present authors [7] were able to obtain, using numerical methods, the approximation $\gamma \approx$ 27.2268. In this paper we determine $\gamma$ exactly as an analytic expression. Moreover, we find a precise asymptotic estimate for the number of planar graphs.

Theorem 1. Let $g_{n}$ be the number of planar graphs on $n$ vertices. Then

$$
g_{n} \sim g \cdot n^{-7 / 2} \gamma^{n} n !
$$

where $g \approx 0.4970043999 \cdot 10^{-5}$ and $\gamma \approx 27.2268777685$ are constants given by explicit analytic expressions.

\footnotetext{
${ }^{\dagger}$ Research supported by Beca Fundació Crèdit Andorrà and Project MTM2004-01728.

${ }^{\ddagger}$ Research supported by Project MTM2004-01728. 
As we show later, for the number $c_{n}$ of connected planar graphs on $n$ vertices, we have the estimate

$$
c_{n} \sim c \cdot n^{-7 / 2} \gamma^{n} n !,
$$

where $\gamma$ is as before and $c \approx 0.4787408907 \cdot 10^{-5}$.

The proof of Theorem 1 is based on singularity analysis of generating functions; see [4,5]. Let $g_{n}, c_{n}$ and $b_{n}$ be as before. As we show in the next section, there are two equations linking the exponential generating functions

$$
B(x)=\sum b_{n} x^{n} / n !, \quad C(x)=\sum c_{n} x^{n} / n !, \quad G(x)=\sum g_{n} x^{n} / n ! .
$$

The dominant singularity of $B(x)$ was determined in [1]; we are able to obtain the dominant singularities of $C(x)$ and $G(x)$, which are both equal to $\rho=\gamma^{-1}$.

In Section 2 we review the preliminaries needed for the proof. In Section 3 we find an explicit expression for the generating function $B(x, y)$ of 2-connected planar graphs counted according to the number of vertices and edges. This is a key technical result in the paper, which allows us to obtain a full bivariate singular expansion of $B(x, y)$ in Lemma 6 . The explicit expression obtained for the function $\beta(x, y, z, w)$ in the statement of Lemma 5 suggests that we are in fact integrating a rational function. This is indeed the case as we explain in Section 3.

In Section 4 we determine expansions of $C(x)$ and $G(x)$ of square-root type at the dominant singularity $\rho$, and then we apply "transfer theorems" $[4,5]$ to obtain estimates for $c_{n}$ and $g_{n}$.

The singular expansions of $C(x)$ and $G(x)$ can be extended to the corresponding bivariate generating functions $C(x, y)$ and $G(x, y)$ near $y=1$. This allows us to prove in Section 5, using perturbation of singularities [5], a normal limit law for the number of edges in random planar graphs. To our knowledge, this problem was first posed in [3].

Theorem 2. Let $X_{n}$ denote the number of edges in a random planar graph with $n$ vertices. Then $X_{n}$ is asymptotically normal and the mean $\mu_{n}$ and variance $\sigma_{n}^{2}$ satisfy

$$
\mu_{n} \sim \kappa n, \quad \sigma_{n}^{2} \sim \lambda n,
$$

where $\kappa \approx 2.2132652385$ and $\lambda \approx 0.4303471697$ are constants given by explicit analytic expressions. The same is true for connected random planar graphs, with the same constants $\kappa$ and $\lambda$.

As a consequence, since $\sigma_{n}=o\left(\mu_{n}\right)$, the number of edges is concentrated around its expected value; that is, for every $\epsilon>0$ we have

$$
\operatorname{Prob}\left\{\left|X_{n}-\kappa n\right|>\epsilon n\right\} \rightarrow 0, \quad \text { as } n \rightarrow \infty .
$$

Previously it had been proved that $\operatorname{Prob}\left\{X_{n}<\alpha n\right\} \rightarrow 0$ and $\operatorname{Prob}\left\{X_{n}>\beta n\right\} \rightarrow 0$, as $n \rightarrow \infty$, for some constants $\alpha$ and $\beta$. The best values achieved so far were $\alpha \approx 1.85$ (shown in [6], improving upon [3]) and $\beta \approx 2.44$ (shown in [2], improving upon [12]). Theorem 2 shows that in fact there is only one constant that matters, namely $\kappa$.

A similar result is the following.

Theorem 3. Let $X_{n}$ denote the number of blocks (2-connected components) in a random connected planar graph with $n$ vertices. Then $X_{n}$ is asymptotically normal and the mean $\mu_{n}$ and variance $\sigma_{n}^{2}$ satisfy

$$
\mu_{n} \sim \zeta n, \quad \sigma_{n}^{2} \sim \zeta n,
$$

where $\zeta \approx 0.0390518027$ is a constant given by an explicit analytic expression.

Next we turn to a different parameter, the number of connected components in random planar graphs.

Theorem 4. Let $X_{n}$ denote the number of connected components in a random planar graph with $n$ vertices. Then asymptotically $X_{n}-1$ is distributed like a Poisson law of parameter $\nu$, where $\nu \approx$ 0.0374393660 is a constant given by an explicit analytic expression.

The above result is an improvement upon what was known so far. It is shown in [10] that $Y_{n}$ is stochastically dominated by $1+Y$, where $Y$ is a Poisson law $P(1)$; Theorem 4 shows that in fact $Y_{n}$ is asymptotically $1+P(\nu)$. The following direct corollary to Theorem 4 is worth mentioning.

Corollary 1. (i) The probability that a random planar graph is connected is asymptotically equal to $e^{-\nu} \approx$ 0.9632528217. (ii) The expected number of components in a random planar graph is asymptotically equal to $1+\nu \approx 1.0374393660$. 
Our last result is the following. Let $\mathcal{A}$ be a family of connected planar graphs, and let $A(x)=$ $\sum A_{n} x^{n} / n$ ! be the corresponding generating function. Assume that the radius of convergence of $A(x)$ is strictly larger than $\rho=\gamma^{-1}$, the radius of convergence of $C(x)$; this is equivalent to saying that $\mathcal{A}$ is exponentially smaller than the family $\mathcal{C}$ of all connected planar graphs.

Theorem 5. Assume $\mathcal{A}$ is a family of connected planar graphs that satisfies the previous condition, and let $X_{n}$ denote the number of connected components that belong to $\mathcal{A}$ in a random planar graph with $n$ vertices. Then asymptotically $X_{n}$ is distributed like a Poisson law of parameter $A(\rho)$.

If we take $\mathcal{A}$ as the family of graphs isomorphic to a fixed connected planar graph $H$ with $n$ vertices, then

$$
A(x)=\frac{n !}{|\operatorname{Aut}(H)|} \cdot \frac{x^{n}}{n !}=\frac{x^{n}}{|\operatorname{Aut}(H)|},
$$

where $\operatorname{Aut}(H)$ is the group of automorphisms of $H$. In particular, if $H$ is a single vertex, we obtain that the number of isolated vertices in a random planar graph tends to a Poisson law $P(\rho)=P\left(\gamma^{-1}\right)$. This proves a conjecture by McDiarmid, Steger and Welsh [10].

As a different application of Theorem 5 we have the following. Recall that $B(x)$ is the generating function of 2-connected planar graphs.

Corollary 2. Let $X_{n}$ denote the number of connected components which are 2-connected in a random planar graph with $n$ vertices. Then $X_{n}$ tend to a Poisson law of parameter $B(\rho) \approx 0.0006837025$.

We wish to emphasize that the approach that eventually has led to the enumeration of planar graphs has a long history. Whitney's theorem [18] guarantees that a 3-connected graph has a unique embedding in the sphere; hence the problem of counting 3-connected graphs is in essence equivalent to counting 3connected maps (planar graphs with a specific embedding). This last problem was solved by Mullin and Schellenberg [11] using the approach developed by Tutte in his seminal papers on counting maps (see, for instance, [15]). The next piece is due to Tutte [16]: a 2-connected graph decomposes uniquely into 3 -connected "components". Tutte's decomposition implies equations connecting the generating functions of 3-connected and 2-connected planar graphs, which were obtained by Walsh [17], using the results of Trakhtenbrot [13]. This was used by Bender, Gao and Wormald [1] to solve the problem of counting 2connected planar graphs; their work is most relevant to us and is in fact the starting point of our research. Finally, the decomposition of connected graphs into 2-connected components, and the decomposition of arbitrary graphs into connected components, imply equations connecting the corresponding generating functions. Analytic methods, together with a certain amount of algebraic manipulation, become then the main ingredients in our solution.

Due to space limitations, some of the proofs have been omitted. A version with full proofs can be found in [8].

Acknowledgements. We are grateful to Philippe Flajolet for his encouragement and useful discussions during our research, to Eric Fusy for his help in simplifying the final expressions in Lemma 5, and to Dominic Welsh for giving us access to an early version of [10].

\section{Preliminaries}

In this section and the rest of the paper we use freely the language and basic results of Analytic Combinatorics, as in the forthcoming book of Flajolet and Sedgewick [5]. In particular, the theory of singular expansions and transfer theorems, and the extensions of the central limit theorem based on perturbation of singularities.

Recall that $g_{n}, c_{n}$ and $b_{n}$ denote, respectively, the number of planar graphs, connected planar graphs, and 2-connected planar graphs on $n$ vertices. The corresponding exponential generating functions are related as follows.

Lemma 1. The series $G(x), C(x)$ and $B(x)$ satisfy the following equations:

$$
G(x)=\exp (C(x)), \quad x C^{\prime}(x)=x \exp \left(B^{\prime}\left(x C^{\prime}(x)\right)\right),
$$

where $C^{\prime}(x)=\mathrm{d} C(x) / \mathrm{d} x$ and $B^{\prime}(x)=\mathrm{d} B(x) / \mathrm{d} x$.

Proof. The first equation is standard, given the fact that a planar graph is a set of connected planar graphs, and the set construction in labelled structures corresponds to taking the exponential of the corresponding exponential generating function. 
The second equation follows from a standard argument on the decomposition of a connected graph into 2 -connected components. Take a connected graph rooted at a vertex $v$; hence the generating function $x C^{\prime}(x)$. Now $v$ belongs to a set of 2-connected components (including single edges), each of them rooted at vertex $v$; hence the term $\exp \left(B^{\prime}\right)$. Finally, in each of the 2-connected components, replace every vertex by a rooted connected graph; this explains the substitution $B^{\prime}\left(x C^{\prime}(x)\right)$. Details can be found, for instance, in $[9$, p. 10].

Let $b_{n, q}$ be the number of 2-connected planar graphs with $n$ vertices and $q$ edges, and let

$$
B(x, y)=\sum b_{n, q} y^{q} \frac{x^{n}}{n !}
$$

be the corresponding bivariate generating function. Notice that $B(x, 1)=B(x)$. The generating functions $C(x, y)$ and $G(x, y)$ are defined analogously. Since the parameter "number of edges" is additive under taking connected and 2-connected components, the previous lemma can be extended as follows.

Lemma 2. The series $G(x, y), C(x, y)$ and $B(x, y)$ satisfy the following equations:

$$
G(x, y)=\exp (C(x, y)), \quad x \frac{\partial}{\partial x} C(x, y)=x \exp \left(\frac{\partial}{\partial x} B\left(x \frac{\partial}{\partial x} C(x, y), y\right)\right) .
$$

In the remaining of the section we recall some results of [1]. Define the series $M(x, y)$ by means of the expression

$$
M(x, y)=x^{2} y^{2}\left(\frac{1}{1+x y}+\frac{1}{1+y}-1-\frac{(1+U)^{2}(1+V)^{2}}{(1+U+V)^{3}}\right),
$$

where $U(x, y)$ and $V(x, y)$ are algebraic functions given by

$$
U=x y(1+V)^{2}, \quad V=y(1+U)^{2} .
$$

In the rest of the paper all logarithms are natural.

Lemma 3 (Bender et al. [1]). We have

$$
\frac{\partial B(x, y)}{\partial y}=\frac{x^{2}}{2}\left(\frac{1+D(x, y)}{1+y}\right)
$$

where $D=D(x, y)$ is defined implicitly by $D(x, 0)=0$ and

$$
\frac{M(x, D)}{2 x^{2} D}-\log \left(\frac{1+D}{1+y}\right)+\frac{x D^{2}}{1+x D}=0 .
$$

Moreover, the coefficients of $D(x, y)$ are nonnegative.

There is a small modification in equation (2.3) with respect to [1]. We must consider the graph consisting of a single edge as being 2-connected, otherwise Lemmas 1 and 2 would not hold. Hence the term of lowest degree in the series $B(x, y)$ is $y x^{2} / 2$.

Let us comment on the equations 2.1 to 2.4. The algebraic generating function $M$ corresponds to (rooted) 3-connected planar maps. The decomposition of a 2-connected graph into 3-connected components implies equations (2.3) and (2.4), The generating function $D(x, y)$ is that of networks, which are special graphs with two distinguished vertices.

In order to state the following Lemma 4 on the singular behaviour of $D(x, y)$ we will need the following notation.

$$
\begin{aligned}
\xi & =\frac{(1+3 t)(1-t)^{3}}{16 t^{3}} \\
Y & =\frac{(1+2 t)}{(1+3 t)(1-t)} \exp \left(-\frac{t^{2}(1-t)\left(18+36 t+5 t^{2}\right)}{2(3+t)(1+2 t)(1+3 t)^{2}}\right)-1 \\
\alpha & =144+592 t+664 t^{2}+135 t^{3}+6 t^{4}-5 t^{5} \\
\beta & =3 t(1+t)\left(400+1808 t+2527 t^{2}+1155 t^{3}+237 t^{4}+17 t^{5}\right) \\
D_{0} & =\frac{3 t^{2}}{(1-t)(1+3 t)} \\
D_{2} & =-\frac{48(1+t)(1+2 t)^{2}\left(18+6 t+t^{2}\right)}{(1+3 t) \beta} \\
D_{3} & =384 t^{3}(1+t)^{2}(1+2 t)^{2}(3+t)^{2} \alpha^{3 / 2} \beta^{-5 / 2}
\end{aligned}
$$


Let us notice a slight change in terminology: functions $\xi$ and $Y$ are denoted, respectively, $x_{0}$ and $y_{0}$ in [1].

A key fact is that for $y$ in a suitable small neighborhood of 1 , the equation $Y(t)=y$ has a unique solution in $t=t(y)$. Then define

$$
R(y)=\xi(t(y))
$$

In the next lemma, $D_{i}(y)$ stands for $D_{i}(t)$ for this value $t(y)$. This applies too to functions $B_{i}(y)$ and $C_{i}(y)$ that we introduce later in the paper.

Lemma 4 (Bender et al. [1]). For fixed $y$ in a small neighborhood of $1, R(y)$ is the unique dominant singularity of $D(x, y)$. Moreover, $D(x, y)$ has a branch-point at $R(y)$, and the singular expansion at $R(y)$ is of the form

$$
D(x, y)=D_{0}(y)+D_{2}(y) X^{2}+D_{3}(y) X^{3}+\mathcal{O}\left(X^{4}\right),
$$

where $X=\sqrt{1-x / R(y)}$ and the $D_{i}(y)$ are as before.

The previous lemma is the key result used in [1] to prove the estimate

$$
b_{n} \sim b \cdot n^{-7 / 2} R^{-n} n !,
$$

where $b$ is a constant and $R=R(1) \approx 0.0381910976$.

\section{Analysis of $B(x, y)$}

From equation (2.3), it follows that

$$
B(x, y)=\frac{x^{2}}{2} \int_{0}^{y} \frac{1+D(x, t)}{1+t} d t .
$$

Our goal is to obtain an expression for $B(x, y)$ as a function of $x, y$ and $D(x, y)$ that, although more complex, does not contain an integral. Recall that the algebraic function $U$ is defined in (2.2), and $D$ is defined in Lemma 3.

Lemma 5. Let $W(x, z)=z(1+U(x, z))$. The generating function $B(x, y)$ of 2-connected planar graphs admits the following expression as a formal power series:

$$
B(x, y)=\beta(x, y, D(x, y), W(x, D(x, y))),
$$

where

$$
\beta(x, y, z, w)=\frac{x^{2}}{2} \beta_{1}(x, y, z)-\frac{x}{4} \beta_{2}(x, z, w),
$$

and

$$
\begin{aligned}
\beta_{1}(x, y, z) & =\frac{z(6 x-2+x z)}{4 x}+(1+z) \log \left(\frac{1+y}{1+z}\right)-\frac{\log (1+z)}{2}+\frac{\log (1+x z)}{2 x^{2}} \\
\beta_{2}(x, z, w) & =\frac{2(1+x)(1+w)\left(z+w^{2}\right)+3(w-z)}{2(1+w)^{2}}-\frac{1}{2 x} \log \left(1+x z+x w+x w^{2}\right) \\
& +\frac{1-4 x}{2 x} \log (1+w)+\frac{1-4 x+2 x^{2}}{4 x} \log \left(\frac{1-x+x z+-x w+x w^{2}}{(1-x)\left(z+w^{2}+1+w\right)}\right) .
\end{aligned}
$$

Proof. From equation (3.1) we obtain

$$
B(x, y)=\frac{x^{2}}{2} \log (1+y)+\frac{x^{2}}{2} \int_{0}^{y} \frac{D(x, t)}{1+t} d t .
$$

We integrate by parts and obtain

$$
\int_{0}^{y} \frac{D(x, t)}{1+t} d t=\log (1+y) D(x, y)-\int_{0}^{y} \log (1+t) \frac{\partial D(x, t)}{\partial t} d t .
$$

From now on $x$ is a fixed value. Now notice that from (2.4) it follows that

$$
\phi(u)=-1+(1+u) \exp \left(-\frac{M(x, u)}{2 x^{2} u}-\frac{x u^{2}}{1+x u}\right),
$$


is an inverse of $D(x, y)$, in the sense that $\phi(D(x, y))=y$. In the last integral we change variables $s=D(x, t)$, so that $t=\phi(s)$. Then

$$
\begin{aligned}
\int_{0}^{y} \log (1+t) \frac{\partial D(x, t)}{\partial t} d t & =\int_{0}^{D(x, y)}\left(\log (1+s)-\frac{x s^{2}}{1+x s}\right) d s \\
& -\int_{0}^{D(x, y)} \frac{M(x, s)}{2 x^{2} s} d s .
\end{aligned}
$$

The first integral has a simple primitive and we are left with an integral involving $M(x, y)$. Summing up we have

$$
B(x, y)=\Theta(x, y, D(x, y))+\frac{1}{4} \int_{0}^{D(x, y)} \frac{M(x, s)}{s} d s,
$$

where $\Theta$ is the elementary function

$$
\Theta(x, y, z)=\frac{x^{2}}{2}\left(z+\frac{1}{2} z^{2}+(1+z) \log \frac{1+y}{1+z}\right)-\frac{x}{2} z+\frac{1}{2} \log (1+x z) .
$$

Now we concentrate on the last integral. From (2.1) and (2.2) it follows that

$$
\int_{0}^{D} \frac{M(x, s)}{s} d s=x \int_{0}^{D} \frac{(1+U)^{2} U}{(1+U+V)^{3}} d s,
$$

where $U$ and $V$ are considered as functions of $x$ and $s$, and where for simplicity we write $D=D(x, y)$ from now on.

From the definition $W(x, s)=s(1+U(x, s))$, we obtain that

$$
\frac{(1+U)^{2} U}{(1+U+V)^{3}}=\frac{W-s}{W(1+W)^{3}} \text {. }
$$

Since $W$ satisfies the equation

$$
x s^{2}+\left(1+2 x W^{2}\right) s+W\left(x W^{3}-1\right)=0,
$$

the functional inverse of $W(x, s)$ with respect to the second variable is equal to

$$
-t^{2}-\frac{1-\sqrt{1+4 x t+4 x t^{2}}}{2 x},
$$

where we use $t$ to denote the new variable.

It follows that

$$
\int_{0}^{D} \frac{W-s}{W(1+W) 3} d s=\int_{0}^{W(x, D)} \frac{\left(Q-1-2 x t-2 x t^{2}\right)(2 Q t-2 t-1)}{2 x t(1+t)^{3} Q} d t,
$$

where for simplicity we write

$$
Q(x, t)=\sqrt{1+4 x t+4 x t^{2}} .
$$

The last integral can be solved explicitly with the help of a computer algebra system such as MAPLE, and we obtain as a primitive the function

$$
\begin{aligned}
& \frac{1-2(t+4 x+4 x t)}{4 x(1+t)^{2}}-\frac{1+2 x(1+t)}{2 x(1+t)^{2}} Q^{3}+\left(2+4 x t+\frac{1+2(t-x-t x)}{4 x(1+t)^{2}}\right) Q+ \\
& \frac{2 x^{2}-4 x+1}{4 x} \log \left(\frac{Q+(1-2 x-2 x t)}{Q-(1-2 x-2 x t)}\right)-\frac{1}{2 x} \log (Q+1+2 x t)+\frac{1-4 x}{2 x} \log (1+t) .
\end{aligned}
$$

Finally we have to replace $t$ for $W(x, D)$ in the previous equation. The expression (3.3) and equation (3.4) imply that

$$
Q(x, W(x, D))=1+2 x\left(D+W(x, D)^{2}\right) .
$$

Hence when replacing $t$ for $W(x, D)$ we obtain an expression in $x, D$ and $W(x, D)$ that is free of square roots. A routine computation, combined with the intermediate equation (3.2), gives the final expression for $B(x, y)$ as claimed. 
From Lemma 4 and 5 we directly obtain a singular expansion for $B(x, y)$ around $R(y)$. The function $R(y)$ is defined in (2.5) and $B_{0}, B_{2}, B_{4}, B_{5}$ are analytic functions of $y$ given in the appendix in [8]. The functions $B_{i}(y)$ can be made explicit in terms of $t$, where $t$ is the unique solution of $Y(t)=y$ in a suitable neighborhood.

Lemma 6. For fixed $y$ in a small neighborhood of 1 , the dominant singularity of $B(x, y)$ is equal to $R(y)$. The singular expansion at $R(y)$ is of the form

$$
B(x, y)=B_{0}(y)+B_{2}(y) X^{2}+B_{4}(y) X^{4}+B_{5}(y) X^{5}+\mathcal{O}\left(X^{6}\right),
$$

where $X=\sqrt{1-x / R(y)}$, and the $B_{i}$ are analytic functions in a neighborhood of 1 .

\section{Asymptotic estimates}

In order to prove Theorem 1, first we need to locate the dominant singularity $\rho=\gamma^{-1}$ of $G(x)$. Since $G(x)=\exp (C(x))$, the functions $G(x)$ and $C(x)$ have the same singularities; hence from now on we concentrate on $C(x)$.

We rewrite the second equation in Lemma 1 as

$$
F(x)=x \exp \left(B^{\prime}(F(x))\right)
$$

where $F(x)=x C^{\prime}(x)$. Notice that the singularities of $B^{\prime}(x)$ and $F(x)$, are the same, respectively, as those of $B(x)$ and $C(x)$. From (4.1) it follows that

$$
\psi(u)=u e^{-B^{\prime}(u)}
$$

is the functional inverse of $F(x)$. The dominant singularity of $\psi$ is the same as that of $B(x)$, which according to Lemma 6 is equal to $R=R(1)$. In order to determine the dominant singularity $\rho$ of $F(x)$, we have to decide which of the following possibilities hold; see Proposition IV.4 in [5] for an explanation.

1. There exists $\tau \in(0, R)$ (necessarily unique) such that $\psi^{\prime}(\tau)=0$. Then $\psi$ ceases to be invertible at $\tau$ and $\rho=\psi(\tau)$.

2. We have $\psi^{\prime}(u) \neq 0$ for all $u \in(0, R)$. Then $\rho=\psi(R)$.

The condition $\psi^{\prime}(\tau)=0$ is equivalent to $B^{\prime \prime}(\tau)=1 / \tau$. Since $B^{\prime \prime}(u)$ is increasing (the series $B(u)$ has positive coefficients) and $1 / u$ is decreasing, we are in case (2) if and only if $B^{\prime \prime}(R)<1 / R$. Next we show that this is the case.

Lemma 7. Let $R$ be as before the radius of convergence of $B(x)$. Then $B^{\prime \prime}(R)<1 / R$.

Proof. Lemma 6 implies that $B^{\prime \prime}(R)=2 B_{4} / R^{2}$ (see (4.3) below). Hence the inequality becomes $2 B_{4}<$ $R$. It holds because $R \approx 0.0381$ and $B_{4} \approx 0.000768$.

We are now ready for the main result.

Proof of Theorem 1. Lemma 7 implies that the dominant singularity of $F(x)$ is at $\rho=\psi(R)$. In order to obtain the singular expansion of $F(x)$ at $\rho$, we have to invert the singular expansion of $\psi(u)$ at $R$.

The expansion of $B^{\prime}(x)$ follows directly by differentiating the one in Lemma 6:

$$
B^{\prime}(x)=-\frac{1}{R}\left(B_{2}+2 B_{4} X^{2}+\frac{5}{2} B_{5} X^{3}\right)+\mathcal{O}\left(X^{4}\right) .
$$

Because of $\psi(x)=x \exp \left(-B^{\prime}(x)\right)$, by functional composition we obtain

$$
\psi(x)=R e^{B_{2} / R}\left(1+\left(\frac{2 B_{4}}{R}-1\right) X^{2}+\frac{5 B_{5}}{2 R} X^{3}\right)+\mathcal{O}\left(X^{4}\right) .
$$

Since we are inverting at the singularity, $F(x)$ also has a singular expansion of square-root type:

$$
F(x)=F_{0}+F_{1} X+F_{2} X^{2}+F_{3} X^{3}+\mathcal{O}\left(X^{4}\right),
$$


with the difference that now $X=\sqrt{1-x / \rho}$. Given that $F(x)$ and $\psi(x)$ are functional inverses, the $F_{i}$ can be found by indeterminate coefficients, and they turn out to be, in terms of $R$ and the $B_{i}$,

$$
F_{0}=R, \quad F_{1}=0, \quad F_{2}=\frac{R^{2}}{2 B_{4}-R}, \quad F_{3}=-\frac{5}{2} B_{5}\left(1-2 B_{4} / R\right)^{-5 / 2} .
$$

The singular expansion of $C(x)$ is obtained by integrating $C^{\prime}(x)=F(x) / x$, and one gets

$$
C(x)=C_{0}+C_{2} X^{2}+C_{4} X^{4}+C_{5} X^{5}+\mathcal{O}\left(X^{6}\right) .
$$

The $C_{i}$, except $C_{0}$, are computed easily in terms of the $F_{i}$ in equation (4.4), and they turn out to be

$$
C_{2}=-F_{0}, \quad C_{4}=-\frac{F_{0}+F_{2}}{2}, \quad C_{5}=-\frac{2}{5} F_{3} .
$$

By singularity analysis, we obtain the estimate

$$
c_{n} \sim c \cdot n^{-7 / 2} \rho^{-n} n !,
$$

where $c=C_{5} / \Gamma(-5 / 2)$.

However, the coefficient $C_{0}=C(\rho)$ is indeterminate after the integration of $F(x) / x$, and is needed later. To compute it, we start by integrating by parts

$$
C(x)=\int_{0}^{x} \frac{F(s)}{s} d s=F(x) \log x-\int_{0}^{x} F^{\prime}(s) \log s d s .
$$

We change variables $t=F(s)$, so that $s=\psi(t)=t e^{-B^{\prime}(t)}$, and the last integral becomes

$$
\int_{0}^{F(x)} \log \psi(t) d t=\int_{0}^{F(x)}\left(\log t-B^{\prime}(t)\right) d t=F(x) \log F(x)-F(x)-B(F(x)) .
$$

Hence

$$
C(x)=F(x) \log x-F(x) \log F(x)+F(x)+B(F(x)) .
$$

Taking into account that $F(\rho)=R$ and $B(R)=B_{0}$, we get

$$
C_{0}=C(\rho)=R \log \rho-R \log R+R+B_{0} .
$$

A simple computation shows that, equivalently,

$$
C_{0}=R+B_{0}+B_{2} .
$$

The final step is simpler since $G(x)=e^{C(x)}$. We apply the exponential function to (4.5) and obtain the singular expansion

$$
G(x)=e^{C_{0}}\left(1+C_{2} X^{2}+\left(C_{4}+\frac{1}{2} C_{2}^{2}\right) X^{4}+C_{5} X^{5}\right)+\mathcal{O}\left(X^{6}\right),
$$

where again $X=\sqrt{1-x / \rho}$. By singularity analysis, we obtain the estimate

$$
g_{n} \sim g \cdot n^{-7 / 2} \rho^{-n} n !,
$$

where $g=e^{C_{0}} c$. Finally, since $\rho=\psi(R)=R e^{-B^{\prime}(R)}$ and $B^{\prime}(R)=-B_{2} / R$, we get

$$
\rho=R e^{B_{2} / R}, \quad \gamma=\rho^{-1}=\frac{1}{R} e^{-B_{2} / R} .
$$

Thus the constants $c, g$ and $\rho$ can be found using the known value of $R$ and the expressions for the $B_{i}$ in the appendix in [8]; the approximate values in the statement have been computed using these expressions.

Notice that the probability that a random planar graph is connected is equal to $c_{n} / g_{n} \sim c / g=e^{-C_{0}}$. This result reappears later in Theorem 4. 


\section{Limit laws}

The proofs of Theorems 2 and 3 are based on bivariate singular expansions and perturbation of singularities. On the contrary, to prove Theorems 4 and 5, univariate asymptotics is enough. To simplify the notation, in this section we denote by $f^{\prime}(x, y)$ the derivative of a bivariate function with respect to $x$.

Proof of Theorem 2. We rewrite the second equation in Lemma 2 as

$$
F(x, y)=x \exp \left(B^{\prime}(F(x, y), y)\right),
$$

where $F(x, y)=x C^{\prime}(x, y)$. It follows that, for $y$ fixed,

$$
\psi(u, y)=u e^{-B^{\prime}(u, y)}
$$

is the functional inverse of $F(x, y)$.

We know from the previous section that $\psi^{\prime}(u, y)$ does not vanish for $y=1$ and $u \in(0, R)$, and that $\rho=\psi(R)$ is the dominant singularity of $F(x)$. Hence by continuity the same is true for $y$ close to 1 , and the dominant singularity of $F(x, y)$ is at

$$
\rho(y)=\psi(R(y), y)=R(y) e^{-B^{\prime}(R(y), y)} .
$$

Given the analytic expressions for the functions involved, the univariate singular expansion of $\psi(x)$ extends to an expansion of $\psi(x, y)$ for $y$ fixed. The same is true then for $F(x, y)$ and $C(x, y)$, and we obtain a bivariate expansion

$$
C(x, y)=C_{0}(y)+C_{2}(y) X^{2}+C_{4}(y) X^{4}+C_{5}(y) X^{5}+\mathcal{O}\left(X^{6}\right),
$$

where now $X=\sqrt{1-x / \rho(y)}$.

Then the so-called quasi-powers theorem [5, Sec. IX.5] implies a limit normal law for the number of edges in random connected planar graphs, with expectation and variance linear in $n$. The constants $\kappa$ and $\lambda$ in the statement of Theorem 2 are given by

$$
\kappa=-\frac{\rho^{\prime}(1)}{\rho(1)}, \quad \lambda=-\frac{\rho^{\prime \prime}(1)}{\rho(1)}-\frac{\rho^{\prime}(1)}{\rho(1)}+\left(\frac{\rho^{\prime}(1)}{\rho(1)}\right)^{2} .
$$

Since $G(x, y)$ and $C(x, y)$ have the same dominant singularities $\rho(y)$, the previous statement also holds for arbitrary planar graphs, with the same values of $\kappa$ and $\lambda$.

In order to determine the parameters exactly, we need only an explicit expression for $\rho(y)$. The expansion (4.3) extends to an expansion of $B^{\prime}(x, y)$, whose constant term is $B^{\prime}(R(y), y)=-B_{2}(y) / R(y)$. Hence from (5.3) it follows that

$$
\rho(y)=R(y) \exp \left(B_{2}(y) / R(y)\right) .
$$

The appendix in [8] contains an explicit expression for $\rho(y)=q(t)$ as a function of $t$. The necessary derivatives are computed as $\rho^{\prime}(y)=q^{\prime}(t) / Y^{\prime}(t)$, and the same goes for $\rho^{\prime \prime}(y)$. The approximate values in the statement have been computed in this way.

Proof of Theorem 4. Let $\nu=C(\rho)=C_{0}$, the evaluation of $C(x)$ at its dominant singularity. For fixed $k$, the generating function of planar graphs with exactly $k$ connected components is

$$
\frac{1}{k !} C(x)^{k} \text {. }
$$

For fixed $k$ we have

$$
\left[x^{k}\right] C(x)^{k} \sim k C_{0}^{k-1}\left[x^{n}\right] C(x) .
$$

Hence the probability that a random planar graphs has exactly $k$ components is asymptotically

$$
\frac{\left[x^{n}\right] C(x) / k !}{\left[x^{n}\right] G(x)} \sim \frac{k C_{0}^{k-1}}{k !} e^{-C_{0}}=\frac{\nu^{k-1}}{(k-1) !} e^{-\nu},
$$

as was to be proved. 


\section{References}

[1] E. A. Bender, Z. Gao, N. C. Wormald, The number of 2-connected labelled planar graphs, Elec. J. Combinatorics 9 (2002), \#43.

[2] N. Bonichon, C. Gavoille, N. Hanusse, D. Poulalhon, G. Schaeffer, Planar Graphs, via Well-Orderly Maps and Trees, Proceedings of WG 2004, Springer LNCS.

[3] A. Denise, M. Vasconcellos, D. J. A. Welsh, The random planar graph, Congr. Numer. 113 (1996), 61-79.

[4] P. Flajolet, A. Odlyzko, Singularity analysis of generating functions, SIAM J. Discrete Math. 3 (1990), 216-240.

[5] P. Flajolet, R. Sedgewick, Analytic Combinatorics (book in preparation), preliminary version available at http://algo.inria.fr/flajolet/Publications

[6] S. Gerke, C. McDiarmid, On the Number of Edges in Random Planar Graphs, Comb. Prob. and Computing 13 (2004), 165-183.

[7] O. Giménez, M. Noy, Estimating the growth constant of labelled planar graphs, in Mathematics and Computer Science III: Algorithms, Trees, Combinatorics and Probabilities (M. Drmota, P. Flajolet, D. Gardy, B. Gittenberger, eds.), Birkhäuser, Basel, 2004, pp. 133-139.

[8] O. Giménez, M. Noy, Asymptotic enumeration and limit laws of planar graphs, math.CO/0501269.

[9] F. Harary, E. Palmer, Graphical Enumeration, Academic Press, New York-London, 1973.

[10] C. McDiarmid, A. Steger, D. Welsh, Random Planar Graphs, J. Combin. Theory Ser. B (to appear).

[11] R. C, Mullin, P. J. Schellenberg, The enumeration of c-nets via quadrangulations, J. Combin. Theory 4 (1968), 259-276.

[12] D. Osthus, H. J. Prömel, A. Taraz, On random planar graphs, the number of planar graphs and their triangulations, J. Combin. Theory Ser. B 88 (2003), 119-134.

[13] B. A. Trakhtenbrot, Towards a theory of non-repeating contact schemes, Trudi Mat. Inst. Akad. Nauk SSSR 51 (1958), 226-269 (in Russian).

[14] G. Turán, On the succinct representation of graphs, Discrete Appl. Math. 8 (1984), 289-294.

[15] W. T. Tutte, A census of planar maps, Canad. J. Math. 15 (1963), 249-271.

[16] W. T. Tutte, Connectivity in graphs, University of Toronto Press, Toronto, 1966.

[17] T. R. S. Walsh, Counting labelled three-connected and homeomorphically irreducible two-connected graphs, J. Combin. Theory Ser. B 32 (1982), 1-11.

[18] H. Whitney, Congruent graphs and the connectivity of graphs, Amer. J. Math. 54 (1932), 150-168. 